\title{
Una nueva mirada sobre la concordancia inesperada en español ${ }^{*}$
}

\author{
A new look on unexpected agreement in Spanish
}

\author{
María Mare \\ Universidad Nacional del Comahue/CONICET \\ mare.purcigliotti@fahu.uncoma.edu.ar \\ ORCID iD: https://orcid.org/0000-0002-9250-2467
}

RESUMEN: En el presente trabajo se describen los diferentes contextos en los que tiene lugar la concordancia inesperada en español (anotenlon) y se discuten las principales propuestas de análisis sobre este fenómeno. La mayoría de los datos recopilados escapan a las explicaciones sugeridas hasta el momento, ya que ilustran que es posible encontrar todas las posibilidades lógicas para la ocurrencia de la $-n$ enclítica y, además, que el copiado/desplazamiento de la morfología de concordancia se produce también con la primera persona del plural, aunque de manera parcial (anotemoslon).

Palabras clave: variación, concordancia, clíticos, pluralidad anómala.

ABSTRACT: In this paper, we describe the different linguistic contexts in which unexpected verbal morphology can be found in Spanish dialects and we discuss the main proposals for this phenomenon. Most of the data we present here, which belong to different dialects, are not explained by the research developed so far, because the examples show that all the logic possibilities for the occurrence of enclitic $-n$ can be observed. Moreover, the copy/displacement of agreement morphology also takes place with the first plural person, although it happens in a partial way.

Keywords: variation, agreement, clitics, unexpected plurality.

\footnotetext{
* Agradezco de manera muy especial a Montse Batllori, Francesc Roca y Enrique Pato, por sus comentarios y sugerencias. También quiero agradecer las observaciones de los evaluadores anónimos.
} 
[A]ndo un poco torpe y disléxico hoy. Cómo se pide a un grupo de personas que te den algo... "demen ustedes" o "denmen ustedes" o "denme ustedes". Parece que es la primera pero no estoy seguro $(<\mathrm{https} / /$ ar.answers.yahoo.com/question/index?qid=20101117092520AAulo2w $>$ ).

\section{INTRODUCCIÓN}

En algunas variedades del español, la ocurrencia de clíticos desencadena distintos fenómenos vinculados con la concordancia. Podemos mencionar, por ejemplo, el caso del se espúreo (se lo dije), que en las variedades del español americano, cuando su referente es plural, puede motivar la ocurrencia de morfología de plural en el clítico acusativo: se los dije a ellos (Bonet, 1995; Company Company, 1992). La ausencia de morfología de número en el clítico se parece extenderse, en muchas variedades, al clítico dativo de tercera persona $l e$, lo que se observa en secuencias como le dije a mis estudiantes $\mathrm{i}_{\mathrm{i}}$ que tomaran apuntes. Otro fenómeno de aparente anticoncordancia se da con la primera persona del plural en construcciones reflexivas y no reflexivas, como se escondíamos en lugar de nos escondíamos (Benito, 2015). En la denominada área mapuche de la Patagonia argentina y chilena el clítico nos aparece sustituido por el clítico los/lo con aspiración de $/ \mathrm{s} /$, como en lo/los escondíamos (Malvestiti, 1993; Acuña y Menegotto, 1998, entre otros). Finalmente, la presencia de clíticos en español puede dar lugar a concordancias "duplicadas" o "desplazadas", cuando son enclíticos, como en ;Larguenlon, nomás! [Güiraldes, Don Segundo Sombras] o ¡Hágalon! (Arregi y Nevins, 2012; Harris, 1995; Harris y Halle, 2005; Kayne, 2010 y Manzini y Savoia, 2011, entre otros).

En este trabajo, nos detenemos en este último fenómeno, particularmente, en las distintas posibilidades que se encuentran en cuanto a su distribución, aspecto que merece una revisión detallada, que no ha sido realizada aún. Nuestro objetivo es describir los distintos contextos en los que se puede reconocer la aparición de morfología de plural inesperada y revisar los problemas que muchos de estos contextos presentan para las aproximaciones previas a la duplicación/desplazamiento del morfema de número y persona en español. Si bien esta presentación es fundamentalmente descriptiva, los análisis previos que recuperamos y el abordaje general de los datos se enmarcan en la Gramática Generativa. Desde este enfoque discutimos los problemas que suponen los datos y plantemos los aspectos que deberían considerarse en la formulación de posibles análisis. El desarrollo de una propuesta formal para este fenómeno excede los objetivos del recorrido que realizamos aquí.

El trabajo se organiza de la siguiente manera. Después de esta introducción, en $\S 2$, presentamos los datos recopilados y proponemos una sistematización de 
estos. En §3, comentamos brevemente las dos posturas que se han adoptado en los estudios previos sobre este fenómeno, a fin de discutir en $\$ 4$ los problemas que los datos relevados en $\S 2$ ofrecen para estos análisis, específicamente, los que involucran la primera persona del plural. Finalmente, en $\S 5$ desarrollamos las consideraciones finales.

\section{REVISIÓN EMPÍRICA}

\subsection{Aspectos generales}

La aparición de morfología de plural en posiciones inesperadas se reconoce en las tres formas verbales que admiten la enclisis: imperativo, infinitivo y gerundio. Como ha sido consignado en la bibliografía, el caso más extendido en las distintas variedades del español es la aparición de $-n$ con imperativos, donde el morfema $-n$ corresponde tanto a la tercera persona como a la segunda, siempre del plural. Las posibilidades básicas para la manifestación de la morfología en posiciones inesperadas son la reduplicación o copiado (1a) y la metátesis o desplazamiento (1b).

(1) a. Váyansen. [Reduplicación o copiado]

b. Váyasen. [Metátesis o desplazamiento]

Cabe señalar, siguiendo la observación de Harris y Halle (2005), que este fenómeno no es estrictamente fonológico, dado que involucra al morfema $-n$ únicamente cuando éste se vincula con el rasgo [PL(URAL)] de los verbos (denlen/delen eso, verbo dar) y no con cualquier instancia del fonema $/ \mathrm{n} /$ (*tenlen/*telen eso > verbo tener). Algo similar sucede con el enclítico, ya que no cualquier secuencia de fonemas que coincida con un clítico desencadena las operaciones de copiado o de desplazamiento. La diferencia entre /lo/ en (2a) y (2b) es que en (2a) encontramos el clítico acusativo, mientras que en ( $2 b)$ tenemos el artículo neutro, que fonológicamente es proclítico del adjetivo mejor.

(2) a. Háganlo mejor $>$ Háganlon mejor/Hágalon mejor

b. Hagan lo mejor $>*$ Hagan lon mejor/*Haga lon mejor

Estas dos observaciones sugieren, de acuerdo con los autores, que la identificación de los elementos que se copian o se desplazan no implica secuencias fonológicas como /nle/ o /nlo/, sino configuraciones morfosintácticas específicas.

Otro aspecto que suele señalarse es que si el elemento enclítico presenta morfología de plural propia, el agregado de $-n$ es imposible. Es decir, una forma como sirvan los da lugar en cualquier dialecto a sirvan los, pero nunca a 
* sirvan'lon o *sirva·lon, y menos aún a la combinación con el morfema de plu$\mathrm{ral} / \mathrm{s} /:$ *sirvanlosn/*sirvanlons. Harris y Halle (2005) indican que este último caso resulta interesante, ya que la explicación de los datos agramaticales no se sigue de una incompatibilidad con un clítico semántica o sintácticamente plural: el clítico se puede corresponderse con un plural, como en ustedes sirvanse, y aun así, participa en el copiado (sirvansen) y el desplazamiento (sirvasen).

(3) a. sirvan $\cdot \operatorname{se} / \operatorname{sirva}(n) \cdot \operatorname{sen}$

b. $\operatorname{sirvan} \cdot \operatorname{los} / * \operatorname{sirva}(\mathrm{n}) \cdot \operatorname{lon} / * \operatorname{sirva} \cdot \operatorname{los}$

La solución que plantean Harris y Halle es que la instanciación fonológica del sufijo plural por defecto /-s/ bloquea el copiado y el desplazamiento por razones fonológicas sobre la formación de codas del español. Es decir, el bloqueo se da por restricciones fonológicas independientes. En §2.3, volvemos sobre las observaciones de los autores con respecto al sufijo plural.

\subsection{Construcciones no flexionadas}

Los datos sobre plurales anómalos en construcciones no flexionadas son mencionados por Harris y Halle (2005) y por Heap y Pato (2012). Los autores observan que tanto los infinitivos como los gerundios, formas verbales que, al igual que el imperativo, admiten elementos enclíticos (venderlo/vendiéndolo), habilitan en algunas variedades del español la presencia de morfología de número y persona después del clítico. Heap y Pato, por ejemplo, recogen ejemplos del español peninsular de diferentes períodos y también dan cuenta de la distribución geográfica del fenómeno. Si bien los datos presentados son sumamente interesantes, no se reconoce una sistematización, algo que resulta relevante para discutir posibles análisis. En nuestra búsqueda, hemos considerado diferentes contextos sintácticos, como construcciones perifrásticas, infinitivos como complemento de preposición, gerundios a nivel de adjunto, etc. Como mostramos en los ejemplos a continuación, todas las construcciones admiten el copiado $^{1}$.

\section{- Perífrasis verbales de infinitivo ${ }^{2}$}

\footnotetext{
${ }^{1} \mathrm{Si}$ bien en este trabajo no nos centramos en revisar la distribución geográfica de estos datos, cabe mencionar que la duplicación en los casos de los infinitivos y gerundios no se extiende a todas las variedades que presentan este fenómeno con el imperativo. La mayoría de los ejemplos que presentamos sobre infinitivos y gerundios corresponden a hablantes colombianos y mexicanos.

${ }^{2}$ En todos los ejemplos mantenemos la grafía original. Asimismo, procuramos brindar información sobre el país de origen, cuando esta se menciona de manera explícita.
} 
(4) a. pueden comprarlon por solo 20.000 colones [Costa Rica] $<$ https://es-la.facebook.com/React-929530410409019/>

b. van a querer matarsen $<$ http://gbooks2.melodysoft.com/app?ID=disturbiorojobogotayDOC= 1386yORDERBY=1>

c. Sin tener que matarsen las unas a las otras $<$ http://aminoapps.com/page/anime-es/2226218/30diaschallenge-dia9-mi-villano-favorito>

\section{- Perífrasis verbales de gerundio}

(5) a. Sin embargo seguimos haciendolon $<\mathrm{http}$ :/hipocritasomostodos.blogspot.com.es/2015/04/aps.html >

b. estarán matandosen en una floristeria o trabajando muy duro $<$ http://doramariagiraldo.blogspot.com.es/p/atrevete-a.html $>$

c. Llevaban tiempo pegandomen innecesariamente

\section{- Construcciones de control y causativas}

(6) a. peliculas donde obliguen a personas a matarsen en un edificio $<$ https://es.answers.yahoo.com/question/index?qid= 20120723172238AAvOKTw>

b. y juraron matarsen cuando volvieran a verse [Tomás González, Abraham entre bandidos]

c. sería muy chistoso ver 2 prist holy intentando matarsen $<$ http://us.battle.net/forums/es/wow/topic/2369679973?page $=2>$

\section{— Infinitivos como complemento de preposición}

(7) a. se quitan el bocado de la boca para darselon a uno $<$ https://bg-bg.facebook.com/tiendasekono $>$

b. lo gracioso es la escusa que utilizan para ponerselon $<$ http://zonaforo.meristation.com/topic/2137487/page-10>

c. aguantan los golpes sin rompersen $<$ https://www.emaze.com/@ALTCLOWW/propiedades-de-los-metales>

d. Creo que los verdaderos amigos se hieren con la verdad para no matarsen con la mentira.

$<$ https://vimeo.com/user47840980>

\section{- Otras construcciones de infinitivo}

(8) a. ampollas pequeñas que al rompersen forman costras [subordinada adverbial]

$<$ http://pospopuli.minsalud.gov.co/pospopuli/Glosario/tabid/738/ FilterID/13680/Default.aspx> 
b. son capaces de hacer cualquier cosa, hasta suicidarsen [complemento de adjetivo]

$<$ http://mensajesnolloveraenel2014.blogspot.com.es/2013/11/a-cuatromiembros-de-la-iglesia-la-luz.html $>$

c. con motivo de cumplirsen los cincuenta años de aquel viaje que marcó su infancia [complemento de nombre] ${ }^{3}$

\section{- Construcciones adverbiales de gerundio}

(9) a. Ellos reaccionan pegandolen

$<$ http://hablajapones.org/foro/japon/escuelas-japonesas-verdadero-ofalso/>

b. Después de dos semanas rompiendosen los cascos $<$ http://www.hozona.org/blog >

c. Escuchaba el ruido de la gente, de los esferos moviendosen, los pupitres arrastrandosen, hojas rompiendosen

$<$ www.calameo.com/books/002937235545338fb2644>

Cabe agregar que Kany (1994 [1945]: 146) menciona datos del español de Costa Rica, en los que los hablantes omiten la marca de infinitivo - $r$ y la única morfología verbal que aparece es la $-n$ después del clítico. Los ejemplos pertinentes son los de (24), extraídos de Kany. Agregamos entre corchetes la forma estándar.

(10) a. Sin que lleguen a sitamen [citarme] lo mandaré a primer grado [Agüero, Romancero tico, p. 49]

b. deben tenesen [tenerse] las niñas enserradas [Agüero, Romancero tico, p. 51]

c. ¿quieren casasen [casarse]? [Agüero, Romancero tico, p. 85]

d. no van a venir después a esquitasen [desquitarse] con naide [Fabián Dobles, Aguas turbias, p. 92].

Finalmente, merece mencionarse el hecho de que el morfema - $n$ después del clítico puede aparecer con verbos psicológicos, como gustar. En este caso, la concordancia no responde a la información del sujeto (oracional), sino a la pluralidad del clítico dativo.

(11) a. A los niños les gusta bañarsen en el río ${ }^{4}$.

b. A los niños no les gusta estarsen quietos.

c. la verdad tengo uno grande y otro pequeño que le[s] gusta pelearsen $<$ http://www.emudesc.com/threads/que-mascota-tienes.7077/page-2>

\footnotetext{
${ }^{3}$ Este ejemplo es de Heap y Pato (2012).

${ }^{4}$ (11a-b) son ejemplos que se mencionan en $<$ http://hispanoteca.eu/Foro-preguntas/ARCHIVO-Foro/Ultracorrecci\%C3\%B3n.htm>.
} 
d. no son $\mathrm{d}[\mathrm{e}]$ esos que les gusta... por lo menos hasta ahora, ni metersen en drogas ni salir mucho por ahí [Garde (Navarra), M, 7?, COSER $]^{5}$

Este parecería ser un nuevo contexto en el que los dativos de los verbos psicológicos del tipo de gustar tienen un comportamiento similar al de los sujetos prototípicos y, por eso, son denominados "sujetos caprichosos" (quirky subjects) (Masullo, 1992: cap. 2) ${ }^{6}$.

En resumen, es relevante destacar que las variedades que admiten la pluralidad anómala con infinitivos y gerundios no muestran restricciones vinculadas con el tipo de estructura subyacente, sino que presentan esta morfología en todos los contextos en los que estas formas no flexionadas aparecen. Señalamos, sin embargo, la necesidad de continuar indagando en las construcciones de control, a fin de reconocer si hay diferencias en cuanto al comportamiento de la pluralidad inesperada cuando el controlador no es el sujeto. El par de ejemplos relevante a tal fin es Me sugirieron leerlo (sujeto plural-controlador singular) y Les/Nos sugirió leerlo (sujeto singular-controlador plural). En las búsquedas realizadas hasta el momento no hemos encontrado ejemplos de concordancias inesperadas a partir de casos como estos o similares, del tipo Me sugirieron leerlon o Nos sugirió leerlon?

Antes de concluir esta sección sobre construcciones no flexionadas, quisiéramos detenernos en un ejemplo muy particular, en el que la presencia inesperada de $-n$ se combina con otro fenómeno frecuente en español: la repetición del clítico en las construcciones que admiten ascenso.

(12) y todas las chicas q te estan insultandoten

$<$ https://loscerroselmusical.wordpress.com/2009/11/17/ $\% \mathrm{C} 2 \% \mathrm{BFjustin}-$ bieber-estas-en-la-lista-pasa/>.

En (12) puede observarse que solamente el clítico posverbal desencadena la ocurrencia del morfema $-n$, mientras que el clítico preverbal aparece sin morfología agregada. Como hemos podido comprobar en las búsquedas en la web y en registros propios, este comportamiento es sistemático en los hablantes que presentan estos dos fenómenos y coincide con la observación general de que

\footnotetext{
5 Ejemplo recopilado por Heap y Pato (2012).

${ }^{6}$ Cabe mencionar que otro fenómeno vinculado con concordancias inesperadas, como es el caso de la concordancia comitativa, presenta este mismo comportamiento (cfr. Mare 2013).

${ }^{7}$ En la bibliografía sobre clíticos (Ordóñez, 2014: §4 y las referencias allí citadas), se han señalado diferencias en cuanto a la posibilidad de ascenso del clítico según el controlador sea el sujeto o el objeto de la oración principal (Lo quiere leer/??Me los sugirió leer), por eso consideramos relevante prestar atención a este aspecto.
} 
los plurales inesperados se dan únicamente con enclíticos y nunca con proclíticos (*lon vendan).

Asimismo, cabe llamar la atención sobre el paralelismo entre la fonología y la morfosintaxis con respecto al comportamiento de los clíticos. Así como en español, en las variedades en las que es posible el desplazamiento del acento primario del verbo al clítico, esto ocurre solamente en posición enclítica y el clítico forma una única palabra fonológica con el verbo (Colantoni y Cuervo, 2013), en las variedades que presentan la pluralidad anómala - como la denominan Heap y Pato (2012) — la ocurrencia de - $n$ después del clítico solo tiene lugar si este elemento es postverbal.

\subsection{La -n enclítica y los plurales parasíticos}

La otra observación realizada en las aproximaciones previas tiene que ver con los enclíticos que presentan morfología de plural. Este punto, con el que estamos totalmente de acuerdo, pide ser observado a la luz de los denominados plurales parasíticos (a mis alumnos se $_{i}$ lo-s $_{i}$ dije). El objetivo es determinar si la imposibilidad de la ocurrencia de $-n$ después del clítico tiene que ver con cuestiones fonológicas o debe analizarse en términos morfosintácticos. En este sentido, la pregunta es si en el contexto de verbo $+s e_{[\mathrm{PL}]}+\mathrm{CL}_{\text {[ACUSATIVO] }}$ el resultado es la terminación en $-s$ (diga(n)senlos a ellos), típica de los plurales parasíticos, o la terminación en $n$ - $($ diga $(n)$ senlon a ellos), típica de la pluralidad anómala. Hemos podido recopilar datos de las dos opciones, sin embargo, este aspecto merece un estudio más sistemático.

(13) a. Eso dejensenlos a esas lokas chismosas $<$ http://www.network54.com/Forum/236893/thread/1232481132/3/ HASTA+QUE+POR+FIN+CAISTE+AHORA+PREPARATE $>$

b. digansenlos a sus viejos $<$ http://www.taringa.net/comunidades/fedee/3742033/Gente-ahora-sihablando-de-enserio.html $>$

(14) Digansenlon a los huerfanos de taxistas, pisteros... $<$ http://www.montevideo.com.uy/contenido/ONU-insta-a-Uruguay-abuscar-alternativas-a-la-privacion-libertad-para-menores-309305?plantilla $=1159>$

En cuanto a los clíticos acusativos plurales que bloquearían la realización del morfema - $n$, es importante señalar que la explicación de este punto no puede reducirse a una cuestión puramente fonológica, tal como se desprende de Harris y Halle, vinculada a la formación de codas en español. Si bien la forma *sirvanlosn es imposible en cuanto a la realización de la coda, la otra opción, * sirvanlons, podría 
ser recuperable bajo los mecanismos de reajuste fonológicos propios del español, como es la introducción de la $e$ epentética: canelón > canelon-e-s; camaleón > camaleón-e-s, etc. Es decir, la combinación - $n s$ puede encontrarse en otras instancias y es recuperable. Sin embargo, en el fenómeno que nos ocupa tanto *sirvanlons como *sirvanlones dan un resultado agramatical.

\subsection{Morfología de primera persona de plural}

Otro de los aspectos mencionados en los estudios previos tiene que ver con el contexto que habilita el copiado o el desplazamiento y hay acuerdo en que este hecho se registra cuando el sujeto refiere a una segunda persona del plural. Sin embargo, hay una posibilidad que no ha recibido suficiente atención en la bibliografía, y a la que, a los fines de esta presentación, denominamos duplicación parcial $(15)^{8}$.

(15) a. se salvó el pellejo, digamoslon [Foro Leproso, ¿Por qué Fabbiani erró el gol?]

b. Por fa chicos demoslen ánimos

$<$ https://twitter.com/naranja_orange/status $>$

En estos ejemplos se observa la presencia inesperada del morfema de segunda/tercera persona de plural $-n$ en el contexto de un verbo que presenta información de primera persona del plural. Al igual que los casos analizados por Harris y Halle (2005) y otros autores, esta construcción solo se da en la variante enclítica (*lon digamos; *len demos), sin embargo, presenta diferencias importantes con respecto al tipo de información que se repite y las posibilidades de ocurrencia del morfema de número y persona en el interior de la palabra fonológica. Como se señala en la bibliografía y como hemos podido registrar (Mare 2015), el morfema de segunda/tercera persona del plural con pronombre enclítico puede aparecer en las variedades no estándar ya duplicado (díganlon), ya únicamente después del clítico (dígalon). En los casos que nos competen aquí, tanto la duplicación total (*digamoslomos) como el desplazamiento $(* d i$ galomos) son agramaticales. Veamos otros datos.

(16) a. Un amigo mio llamado Marcelo muy probablemente se prenda. Anotemoslon. [ARG]

b. la pizza a la piedra es una bosta, digamoslon, es una galleta con queso $[\mathrm{ARG}]$

$<$ http://www.fotolog.com/ivancine/7668080/>

\footnotetext{
${ }^{8}$ Minkoff (1993) considera construcciones con la primera persona del plural en el español del Caribe, variedad en la que observa el desplazamiento de la $-s$ de $-m o s$ (demoles por demosle).
} 
c. hagamoslon el domingo el asadito [ARG]

$<\mathrm{http}$ ://inmorriswetrust.foroactivo.com/t1641-jajaj-pero-que-hijo-deputa $>$

d. que sí, que la fiesta hagamoslan

$<$ http://sobrelatexturadealgunascuestiones.blogspot.com.es/2011/10/ los-del-segundo.html>

e. Al menos digamoslen luciano, jefe no más $<$ http://los-historicos.blogspot.com.es/2006/04/jefe-de-queposteadopor-ciro.html>

f. A uno no le gusta! peguemoslen! $<$ https://www.youtube.com/watch?v=ZQD7PUlgZg8>

g. Por fa chicos demoslen ánimos $<$ https://twitter.com/naranja_orange/status $>$

h. ya fue demoselon a niubel $<$ https://es-la.facebook.com/Tomi.Videos/>

Como puede verse, los clíticos que aparecen en estos casos son los mismos que en el copiado y desplazamiento de la segunda/tercera persona del plural, $i$. e., acusativo masculino (16a-c), acusativo femenino (16d) y dativo (16e-g). Además, es posible la combinación de dos clíticos (16h), que podría dar lugar a seis combinaciones lógicas. Sin embargo, los resultados son agramaticales en la mayoría de los casos. Concretamente, la repetición del morfema -mos y su desplazamiento fuera de su posición de base dan resultados inaceptables, algo que no sucede con $-n$ (18).

(17) a. Digamoslon/*Digamoslomos

b. Demoslen el libro/*Demoslemos el libro

c. Demoselon

(18) a. venda-se-n-lo/ *venda-se-mos-lo [V + CL + n/mos + CL]

b. venda-se-lo-n/*venda-se-lo-mos $[\mathrm{V}+\mathrm{CL}+\mathrm{CL}+n / m o s]$

c. venda-se-n-lo-n/ *venda-se-mos-lo-mos $[\mathrm{V}+\mathrm{CL}+\mathrm{n} / \mathrm{mos}+\mathrm{CL}+n /$ mos]

d. venda-n-se-n-lo/ *venda-mos-se-mos-lo $[\mathrm{V}+n / m o s+\mathrm{CL}+n / m o s+$ CL]

e. venda-n-se-lo-n/ *venda-mos-se-lo-mos $[\mathrm{V}+n / \operatorname{mos}+\mathrm{CL}+\mathrm{CL}+n /$ mos]

f. venda-n-se-n-lo-n/ *venda-mos-se-mos-lo-mos $[\mathrm{V}+n / m o s+\mathrm{CL}+n$ / mos $+\mathrm{CL}+n / m o s]$

El hecho de que la primera persona aparezca en relación con morfemas vinculados prototípicamente a la tercera persona no es un fenómeno aislado en español. En distintas variedades rurales se registra el uso de clíticos de tercera persona en lugar de la forma esperable de primera. Según la variedad, podemos encontrar el clítico acusativo lo o la forma se (para la sustitución por se, v. Benito, 2015). 
(19) a. si es que existe tal lanzallamas muestra fotos videos etc. callanos y si no tienes pruebas es que te gusta hacerlon emocionar y luego decilucionarnos $<$ http://zonaforo.meristation.com/topic/738472/page-2>

b. asi q pongamoslon las pilas $<$ http://juguitodenaranjaa.blogspot.com.es/2007/08/estoy-calientesband.html>

(20) a. El novio le decía al padre: "Mira, hamos pensao de casarse, ¿a usté qué le parece? [Lucena del Cid, Castellón, COSER 1308]

b. Sí, se fuimos por la mañana y volvimos por la noche (Palacios Blan$\cos \mathrm{I} 2$, MU 3111)

\subsection{Agrupamiento de clíticos}

Finalmente, merece una mención especial la observación con respecto a las posibilidades de combinación cuando son dos los elementos enclíticos, ya que este punto ha sido fundamental para las propuestas de análisis del fenómeno. La combinación de dos elementos enclíticos da lugar a seis posibilidades lógicas, como destacan Harris y Halle (2005) y Arregi y Nevins (2012 y 2015). Esto autores indican que de las seis posibilidades que mostramos en el Cuadro 1, hay dos que no serían posibles: desplazamiento, desplazamiento (*vendamelón) y copiado, desplazamiento (*vendanmelon). Volvemos sobre este punto en $\S 3$.

\begin{tabular}{|l|l|}
\hline \multicolumn{1}{|c|}{ Operación } & \multicolumn{1}{c|}{ Ejemplo } \\
\hline Desplazamiento & venda-me-n-lo \\
\hline Desplazamiento, desplazamiento & venda-me-lo- $n$ \\
\hline Desplazamiento, copiado & venda-me- $n$-lo- $n$ \\
\hline Copiado & venda- $n-m e-n-l o$ \\
\hline Copiado, desplazamiento & venda- $n-m e-l o-n$ \\
\hline Copiado, copiado & venda- $n-m e-n-l o-n$ \\
\hline
\end{tabular}

CUADro 1.-Posibilidad de ocurrencia de $-n$ con clíticos

Sin embargo, las posibilidades que el análisis de Arregi y Nevins permiten descartar, son opciones válidas que se encuentran en otras variedades del español (Belloro, 2015: 76), como muestran los ejemplos de (21) y (22).

\footnotetext{
${ }^{9}$ Los ejemplos de (20a) y de (20b) fueron extraídos de De Benito (2015: 96 y 116, respectivamente).
} 
(21) a. Pongaselón completo, por lo menos ${ }^{10}$ [desplazamiento, desplazamiento]

b. asi que chicas haga selon antes de que se arepienta $<\mathrm{http}$ //www.forofantasiasmiguel.com/tm.aspx?m=1491209ympage $>$

c. Dejeselon a los presos ahí dentro [Costa Rica] $<$ http://www.blogtelopia.com/un-buscador-de-personas-eninternet.html>

d. El dolor dejeselon pa uds $<$ http://ask.fm/KamiiOkampo/answers/111563168179>

e. No lo intenten en casa dejeselon a los profesionales $<\mathrm{https}$ ://www.youtube.com/all_comments?gl=SNyhl=fryv $>$

f. ese nombre dejeselon para los equipos grandes $<$ https://m.login.connect.facebook.com/.../a.../10153894467894976/?...3...>

g. todo dejeselon en manos de Dios $<$ https://www.pixgram.me/aprd5/media/1290945476799825902_ $446448467>$

(22) a. Diganmelon a mí [copiado, desplazamiento] ${ }^{11}$ $<$ http://www.taringa.net/ElPapadelagente/mi/3uztI $>$

b. los cupones obligan a la gente a no trabajar... mira denselón a las madres trabajadoras [Puerto Rico]

c. va a llegar muy lejos!!! prestenmelon para mi banda, jajaja. [Argentina] $<$ https://www.youtube.com/watch?v=ovo9Fg 8 b 7 aAylist $=$ PL8E79F7248939EF04>

d. No me acuerdo del gol del piojo, muestrenmelon [Argentina] $<$ https://larefundacion.com/2015/07/14/argentina-primer-mundopresentacion-y-arqueros/>

e. si lo venden, vendanselon a Chacarita [Argentina] $<\mathrm{http}: / /$ www.fotolog.com/mati_racing95/73529017/>

f. dejenselon como Chaplin (el bigote) [Argentina] $<$ http://elviejovizcacha.blogspot.com.es/2009/12/afeita-anibal.html>

g. si no saben dejenselon a personas que si sepan $<$ www.portalxd.com/tema/una-pregunta-119684/>

Incluso, también es posible recuperar ejemplos de formas que podrían considerarse de (1) desplazamiento, desplazamiento o de (2) copiado, desplazamiento, según cómo se interprete la ausencia de la $-n$ vinculada a la base. Nos referimos a casos en los que el clítico más cercano al verbo es nos. Estos datos también se descartan en el abordaje que hacen Halle y Marantz (1994: 287).

(23) a. por favor, si no los quieren criar denoslon para los que SI los queremos...

$<$ http://www.fenix951.com.ar/nuevo_2013/noticia.php?id=14385>

\footnotetext{
${ }^{10}$ Ejemplo de Belloro (2015: 76).

11 Kany (1994 [1945]: 145) menciona los siguientes casos de copiado-desplazamiento: ¡Mirenmelón al doctor! (Fray Mocho, p. 155, ARG); ;Mirenmelán a la presumida! (Lynch, Palo verde, p. 143. ARG).
} 
b. No es más gratis el juego? Tenemos que comprar las fichas si o no? $\mathrm{Si}$ es asi diganoslon y listo

$<$ https://www.facebook.com/boyaapokersp/posts/260122394068725>

\subsection{Síntesis}

En resumen, en este apartado hemos procurado presentar una variedad de ejemplos vinculados a la ocurrencia de morfología inesperada. En este sentido, buscamos, además, sistematizar algunas cuestiones que, consideramos, pueden ser importantes para avanzar con la investigación de este fenómeno. Los tres puntos centrales del recorrido que hemos presentado tienen que ver con:

(1) La ocurrencia de $-n$ en todos los contextos en los que es posible tener infinitivos y gerundios. Si bien esto ha sido señalado en diferentes textos (Harris, 1995; Harris y Halle, 2005; Heap y Pato, 2012), en ningún caso se ha presentado una sistematización tendiente a determinar si cualquier construcción admite la aparición de $-n$. Este aspecto es fundamental para plantear un análisis, principalmente, cuando se ha señalado que ciertos contextos bloquean la ocurrencia de $-n$. Aquí vimos que este fenómeno es independiente de la configuración sintáctica subyacente a la forma no flexionada, $i$. e., encontramos duplicación tanto en perífrasis verbales, como en construcciones de control, adjuntos, complemento de preposición, etc.

(2) El copiado no se limita a la concordancia de segunda/tercera persona del plural, sino que también se da con la primera persona del plural. En estos casos, es posible encontrar copiado (digamoslon), no desplazamiento (*digalomos) (ver, sin embargo, §4), y como señalamos, el copiado es parcial: solamente se repite la información vinculada con la pluralidad.

(3) Por último, presentamos evidencia empírica para cada una de las seis posibilidades lógicas que tienen lugar cuando los elementos enclíticos son dos. Es decir, no hay restricciones con respecto al lugar/a los lugares en los que puede aparecer el morfema - $n$, aunque sí concordamos con la observación de que las posibilidades están sujetas a variación.

\section{APROXIMACIONES PREVIAS}

Harris y Halle cierran su artículo apelando a cabezas y manos más jóvenes para continuar la discusión de los datos sobre la concordancia inesperada (2005: 219). De la presente sección se sigue que su pedido ha convocado el interés sobre el tema y nosotros esperamos sumar nuevas líneas que permitan avanzar con el estudio de este fenómeno. A tal fin, en el apartado anterior intentamos 
presentar los datos de la duplicación/desplazamiento de manera completa - o lo más completa que nos permite nuestra comprensión- y sistematizada. En adelante, revisaremos las principales discusiones sobre este fenómeno (v. §3.1 y §3.2) y los problemas que los datos que hemos relevado aquí suponen para cada una de ellas (v. $\$ 3.3$ y $§ 4)$.

Las propuestas de análisis sobre la concordancia inesperada en español que detallamos aquí pueden dividirse según aborden el fenómeno como el efecto de procesos postsintácticos (Harris y Halle, 2005; Arregi y Nevins, 2012 y 2015) o como el resultado de operaciones propias de la sintaxis (Kayne, 2010). Como veremos en $\S 3.3$, estos abordajes discuten entre sí y buscan superar los problemas que observan en los análisis precedentes.

\subsection{Propuestas postsintácticas}

Sin bien el fenómeno que nos compete es mencionado en propuestas teóricas previas (Harris, 1991; Minkoff, 1993), el artículo de Harris y Halle (2005) marca el inicio de una discusión empírica y teóricamente relevante. Los autores distinguen entre la reduplicación o copiado (váyansen) y la metátesis o desplazamiento (váyasen). Su propuesta, retomada en Halle (2008), supone la aplicación de restricciones fonológicas sobre la configuración morfosintáctica (no sobre fonemas). Las operaciones que proponen, dentro del marco de la Morfología Distribuida (Halle y Marantz, 1994), son la de copiado [Kopy $]^{12}$ para explicar la reduplicación total o parcial, y Metátesis de flexión verbal [Verbal Inflection Metathesis (VIM)], para dar cuenta de casos de desplazamiento, como váyasen, en los que se observa una alteración en el orden. $\mathrm{Su}$ análisis supone procesos morfofonológicos, $i$. e., que tienen lugar en el componente postsintáctico.

La configuración que ofrecería la sintaxis permite reconocer una cadena formada por el tema verbal, el morfema de concordancia y el clítico. Para esta cadena, Harris y Halle proponen una regla de reajuste que consiste, en primer lugar, en el encorchetamiento de /n/ con el clítico, para indicar el copiado de ese bloque, y, en segundo lugar, en la inserción de paréntesis angulares para dar cuenta de qué parte de ese bloque no se pronuncia. En (24) presentamos de manera simplificada la propuesta de análisis.

(24) a. vendanlon [copiado] venda $[\mathrm{n}<\cdot \mathrm{lo}] \rightarrow$ venda-n $\cdot \mathrm{lo}-\mathrm{n} \cdot l o=$ vendan $\cdot$ lon

b. vendalon [desplazamiento] venda $[\mathrm{n} \gg \cdot \cdot \mathrm{lo}] \rightarrow$ venda- $n \cdot \operatorname{lo}-\mathrm{n} \cdot l o=$ venda $\cdot$ lon

\footnotetext{
${ }^{12}$ Los autores emplean Kopy para referir al copiado postsintáctico, mientras que Copy se utiliza para el copiado en la sintaxis.
} 
Arregi y Nevins (2012 y 2015) también buscan una explicación postsintáctica a este fenómeno. Aunque difieren con Harris y Halle en el tipo de operación de reordenamiento responsable de la manifestación de la concordancia inesperada, consideran que el espíritu de su análisis, al que denominan "reduplicación generalizada", permite entender la metátesis como un caso de reduplicación parcial en la morfofonología, que a su vez deriva del hecho de que la metátesis morfológica y el doblado son procesos estrechamente relacionados que pueden alternar para satisfacer restricciones de linealidad. Bajo este principio, es posible explicar diversos fenómenos que involucran el orden lineal en distintas lenguas ${ }^{13}$.

El foco del análisis de estos autores no solo es explicar el copiado y el desplazamiento, sino también dar cuenta de las restricciones a las que están sujetos ambos procesos. Tales restricciones serían también postsintácticas y se vincularían al agrupamiento de clíticos (clitic clusters). Concretamente, proponen que en la variedad estándar $-n$ es un nodo de concordancia que no se reanaliza morfológicamente con los elementos enclíticos, mientras que en las variedades que admiten el copiado y el desplazamiento, $-n$ se convierte en un clítico. Cuando esto sucede, $-n$ participa también en el agrupamiento de clíticos, en el que el requerimiento de no-inicialidad de $-n$ desencadena el desplazamiento/copiado ${ }^{14}$.

En cuanto a los casos en los que aparecen dos clíticos (venda- $n_{\mathrm{CL}^{-}}-m e_{\mathrm{CL}^{-}}$ $\left.l o_{\mathrm{CL}}\right)$, los autores proponen un primer ciclo de encorchetamiento entre $-n$ y el clítico más cercano y un segundo ciclo de encorchetamiento entre este objeto complejo, i. e. $\left[{ }_{\mathrm{CL}} n_{\mathrm{CL}}><m e_{\mathrm{CL}}\right]$, con el clítico más externo. El clítico externo lo puede reencorchetarse con todo el complejo o bien solo con el clítico que está a su izquierda, i. e. $m e_{\mathrm{CL}}$. En el primer caso, el resultado es vendamenlo, sin desplazamiento de $-n$, ya que cumple con la restricción de no-inicialidad; mientras que el segundo encorchetamiento da vendamelon, con desplazamiento para respetar la restricción mencionada.

Como mencionamos en $\$ 2.5$, la aparición de dos elementos enclíticos da lugar a seis variantes que los autores dividen en dos grupos según el morfema de concordancia $-n$ aparezca in situ (25) o no (26).

(25) a. vendanmenlo [copiado]

b. vendanmelon

c. vendanmenlon

${ }^{13}$ De hecho, Arregi y Nevins (2012: cap. 5) examinan datos del español, el vasco, el irlandés antiguo, lituano, italiano, rumano, etc.

${ }^{14}$ Los autores plantean que tanto el copiado como el desplazamiento tienen lugar de manera cíclica y, en términos descriptivos, respetan el principio de Wackernagel: $-n$ está sujeto a requerimientos de segunda posición dentro del bloque de clíticos (clitic cluster). Un bloque de clíticos es un dominio postsintáctico definido jerárquicamente, que tiene su propio acento final (secundario), de acuerdo a lo señalado por diferentes autores (Roca, 1986; Harris, 1991; Colantoni y Cuervo, 2013). 
(26) a. vendamenlo [desplazamiento]

b. vendamelon

c. vendamenlon

Ahora bien, siguiendo lo señalado por Harris y Halle, no todos los hablantes aceptan todas las posibilidades. Los autores plantean, entonces, que la mesoclisis está sujeta a tres restricciones generales. La primera tiene que ver con la restricción del morfema de concordancia de saltar por encima de más de un clítico. De esta forma, se descartaría *venda-me-lo- $n$ (desplazamiento-desplazamiento) y *venda-n-me-lo-n (copiado-desplazamiento). La segunda se refiere a que la jerarquía de persona rige qué clíticos pueden participar de la mesoclisis. Algunos hablantes solo aceptan la mesoclisis para los clíticos que no tienen información de tercera persona (me: $1 \mathrm{SG}$ y $s e$ : reflexivo), mientras que otros también admiten los clíticos con información de tercera persona $(l o, l a, l e)$. Finalmente, hay una prohibición universal sobre la mesoclisis con clíticos plurales y esto descartaría casos como los que vimos anteriormente (*vendanlosn, *vendanlons) ${ }^{15}$.

\subsection{Propuesta sintáctica}

Kayne (2010) propone un abordaje en el que las operaciones y los principios involucrados en lo que se denomina formación de palabras son esencialmente los mismos que los involucrados en la sintaxis. Esto significa que todos los morfemas se combinan en la sintaxis vía ensamble (Merge). Un análisis estrictamente sintáctico permite derivar directamente la agramaticalidad de * $\mathrm{Ha}$ gan lon mejor contra Haganlon mejor y la imposibilidad de la forma *tenlen contra denlen. Básicamente, plantea que ambos casos son agramaticales, porque los artículos definidos no presentan tanta libertad de movimiento en la sintaxis como los clíticos y porque la sintaxis no puede manipular partes de morfemas.

El autor observa, además, que los clíticos que participan de manera más productiva en el copiado y en el desplazamiento coinciden con el orden predominante de los clíticos del español cuando coaparecen.

$$
\begin{aligned}
& \text { (27) a. se } \\
& \text { b. se, me } \\
& \text { c. se, me, le } \\
& \text { d. se, me, le, lo, la }
\end{aligned}
$$

De acuerdo con Kayne, el análisis debe captar el vínculo que existe entre el orden de los clíticos en español y la distribución del clítico seguido de - $n$, fenó-

${ }^{15}$ Remitimos a Arregi y Nevins (2012 y 2015) para mayores especificaciones. 
meno transdialectal. A partir de estudios previos sobre el comportamiento de los clíticos en italiano y otras lenguas romances (Kayne, 1992), postula que en la forma flexionada, hay un auxiliar silente cuya presencia licencia la posición de lo, inesperada en relación al resto de las construcciones del italiano (Non far-lo 'no hacer.inf.3AC' = 'no lo hagas', pero Non lo fare, en imperativos negados). non lo AUX fare

En el caso de las variedades del español estudiadas, el segundo morfema - $n$ (denle- $n$ ) compartiría con el clítico del italiano la propiedad de ser licenciado por un auxiliar silente, concretamente, una proyección de AGR (concordancia). Así, cada ocurrencia de - $n$ en una posición inesperada supondría una proyección AGR en la sintaxis, por encima de la proyección propia de la variedad estándar.

(29) a. denlen eso= de AGR n le AGR n eso [copiado]

b. denmenlon= de AGR n me AGR n lo AGR n [copiado]

c. demen eso $=$ de me AGR n eso ${ }^{16}$ [desplazamiento]

\subsection{Comentarios las propuestas}

En los subapartados anteriores, revisamos tres propuestas de análisis para las concordancias inesperadas en español. Las dos primeras consideran que se trata de un fenómeno postsintáctico de reordenamiento lineal (inversión de orden y borrado para Harris y Halle; recategorización y no-inicialidad de - $n$ para Arregi y Nevins), mientras que la última sostiene que se trata de un fenómeno que se sigue de las leyes propias de la sintaxis (jerarquía de clíticos, generación de nodos de concordancia). En este recorrido, el texto precursor es el de Harris y Halle, en el que Kayne reconoce diversos problemas. Arregi y Nevins retoman la discusión y, a su vez, presentan sus críticas al análisis de Kayne. Veamos los aspectos que señalan.

La primera crítica de Kayne (2010) con respecto a la propuesta de Harris y Halle se refiere a la morfología distribuida en general, marco al que considera redundante. En este caso en particular, la redundancia vendría dada porque la sintaxis presenta un orden entre palabras y morfemas y luego la morfología interviene también para establecer un orden. La segunda crítica de este autor se refiere a la falta de restricción de la propuesta de Harris y Halle, ya que la metátesis podría intervenir sobre la estructura sintáctica de cualquier otro par y

\footnotetext{
${ }^{16}$ Kayne no explicita el análisis que mostramos en (29c), como sí lo hace con los dos casos anteriores.
} 
no lo hace (*avéndlo, en el que la metátesis se aplica sobre la vocal temática $a$ y la raíz vend-; *vendloan, metátesis sobre vocal temática y clítico). De acuerdo con Kayne, la formalización de Harris y Halle no evitaría los casos agramaticales mencionados y por eso su propuesta es explícita con respecto al rol del nodo AGR. Por otra parte, agrega que la propuesta de Harris y Halle no capta una generalización con respecto a qué clíticos participan de manera más productiva en el fenómeno en cuestión y a su relación con el ordenamiento general de los clíticos en el español general.

Arregi y Nevins (2015), por su parte, revisan la propuesta de Kayne y señalan que, si bien es fundamental captar las restricciones con respecto a esta construcción, algo que el autor explicita, hay dos problemas que surgen de este análisis. En primer lugar, esta aproximación da cuenta del orden de los clíticos, de los lugares en los que aparece - $n$ y permite formalizar la variación; sin embargo, los casos de desplazamiento como demen eso, permitirían considerar la posibilidad de que no se genere ningún nodo AGR (ustedes *deme/denme eso) y no hay nada en el sistema de Kayne que prevenga esto. Al contrario, el análisis de este caso habilita esa posibilidad, ya que AGR no se proyecta en su posición prototípica. Tampoco parece restringirse la generación de muchos nodos AGR (de- AGR $n$ AGR $n$ me AGR $n=*$ dennmen). La crítica de Arregi y Nevins al agregado de nodos AGR se debe, además, al hecho de que se pierde una generalización relevante: puede haber como máximo un morfema - $n$ más que el número de elementos enclíticos. Es decir, si hay un clítico, puede haber como máximos dos - $n$ (copiado: venda-n-lo-n) pero no más (*venda-n-n-lo-n).

Cabe señalar, además, que si bien en Harris y Halle se menciona la aparición de concordancias inesperadas con infinitivos (quieren venderlon) y gerundios (siguen vendiéndolon), ninguna de las propuestas contempla estos datos para proponer su análisis. Una aproximación como la de Kayne, en la que se pueden agregar nodos AGR en la sintaxis podría extenderse a estos contextos. Sin embargo, los abordajes de Harris y Halle y de Arregi y Nevins se enfrentan con serias dificultades para explicar estos datos que no suponen una relación de adyacencia evidente entre el morfema de número y persona de plural y el clítico. Si bien las concordancias inesperadas con imperativos son las más productivas en las diferentes variedades del español, sería deseable que un análisis para estas pudiera extenderse también a aquellas variedades que admiten este fenómeno con infinitivos y gerundios.

Otra cuestión que resultaría problemática, fundamentalmente para Arregi y Nevins, es la existencia de datos de combinación de clíticos que muestran (1) desplazamiento, desplazamiento (vendamelon) y (2) copiado, desplazamiento (vendanmelon). Como vimos en los ejemplos de (34) y (35), ambas configuraciones son posibles, sin embargo, el análisis de Arregi y Nevins las descartaría (v. §3.1). 
El otro conjunto de datos problemáticos para los tres análisis es el que involucra a la primera persona del plural, caso que no aparece registrado en ninguno de los abordajes. En este aspecto nos detendremos en el próximo apartado.

\section{EL CASO DE LA PRIMERA PERSONA DE PLURAL}

En el apartado §2, presentamos una serie de contextos en los que se observan los plurales anómalos, muchos de los cuales no han sido considerados en los análisis que expusimos en la sección anterior. En términos estrictamente intuitivos, lo que parece estar sucediendo en todos esos casos es que los hablantes interpretan el clítico como parte del verbo y dado que estas formas no son asimilables a las terminaciones verbales prototípicas, agregan el morfema de concordancia verbal. Esta idea está presente en la descripción que hacen Alvar y Pottier (1993: 193-194) y Kany (1994 [1945]: 144). Visto así, este fenómeno no se aleja de otro que se observa con el pretérito perfecto de la segunda persona del singular (cantaste), a la que muchos hablantes agregan el morfema -s (cantaste-s), asociado en todos los demás tiempos verbales con la información de segunda persona (canta-s/cantará-s/cantaría-s/cantaba-s, etc). Parecería ser que en ambos casos, hay algún elemento que "invisibiliza" la manifestación de la morfología de concordancia y esta debe volver a "aparecer" ${ }^{17}$. Consideramos que, idealmente, esta intuición debe ser captada por los análisis propuestos, algo que está relativamente presente en el espíritu del análisis de Kayne (2010) y en la propuesta de Harris $(1995)^{18}$, que no hemos desarrollado aquí.

Ahora bien, el caso de la ocurrencia de $-n$ con la primera persona del plural es claramente problemático para cualquier propuesta que suponga el copiado o el desplazamiento de un morfema, ya que sería esperable, si así fuera, que el morfema que aparece después del clítico fuera -mos y no $-n$. Otro de los aspectos que no admite una explicación dentro de estos análisis es el hecho de que

\footnotetext{
${ }^{17}$ Alvar y Pottier (1993: 193-194) vinculan la ocurrencia anómala de la $-s$ y de la $-n$ en español con un fenómeno observado en el judeo-español de Marruecos: "[C]uando la forma verbal va seguida de un pronombre, se pierde la $-s$ desinencial y se añade otra analógica al pronombre (cobižemes 'me cobijes'; cobriésemes 'me cubrieses'). (...) [A]l tornarse un pronombre enclítico $($ cobižes $+m e)$, la $-s$ caracterizadora de la persona tú quedaba en posición interior, con lo que el verbo (cobižesme) perdía el rasgo externo de la persona, entonces se tomó una $-s$ que sirviera para caracterizarla (*cobižesmes), igual que en muchas hablas vulgares la $-n$ (índice de ellos) se incorpora al pronombre enclítico como marca de otro modo no percibida (sientensen o, por disimilación, sientesen). Una vez formado el analógico *cobižesmes, las dos eses (s...s) se consideraron redundantes, e igual que -stes $>$-tes, tras una analogía bien parecida a la que estamos comentando, -smes >-mes."

${ }^{18}$ Harris (1995) propone un reanálisis morfosintáctico. Remitimos a su trabajo para una revisión detallada de esta propuesta.
} 
en ninguna variedad puede estar ausente el morfema -mos de su posición original, $i$. e., posterior a la base verbal, algo que sí sucede con el morfema - $n$ (ver los datos de 17 y 18).

\begin{tabular}{|l|l|l|}
\hline PERSONA & COPIADO & DESPLAZAMIENTO \\
\hline \multirow{2}{*}{ 2/3PL } & vendanlon & vendalon \\
\cline { 2 - 3 } & denlen & delen \\
\hline \multirow{2}{*}{$1 \mathrm{PL}$} & vendamoslon/*vendamoslomos & $*$ vendalon/*vendalomos \\
\cline { 2 - 3 } & demoslen/*demoslemos & $*$ delen/*delemos \\
\hline
\end{tabular}

CUADro 2.-Copiado y desplazamiento con 2/3PL y $1 \mathrm{PL}$

La propuesta de análisis de Harris y Halle, basada en la regla de reajuste que expusimos en $\$ 3.1$ genera todas las construcciones marcadas como agramaticales en el Cuadro 2 y no genera la alternativa gramatical.

(30) a. vendamoslon [copiado]

Venda[mos $<\cdot 10] \rightarrow$ venda-mos $\cdot 10-m o s \cdot l o=*$ vendamoslomos

b. vendamoslon [desplazamiento]

Venda[mos〉 $\langle\cdot \mathrm{lo}] \rightarrow$ venda-mos $\cdot 10-m o s \cdot l o=*$ vendalomos

Por su parte, el análisis de Kayne (2010) basado en una proyección AGR que licencia la ocurrencia de $-n$, resulta más intuitivo con respecto a lo que parece que hacen los hablantes al agregar este elemento, pero no salva el problema con respecto al tipo de información que tendría AGR. En su propuesta, cada nodo AGR que se agrega, repite la información del nodo "original", por lo que nuevamente, se esperaría que se generaran las formas agramaticales y no se generaran las gramaticales.

Finalmente, el análisis de Arregi y Nevins (2012 y 2015) en términos de reduplicación generalizada, que supone el cambio de categoría del nodo AGR, convirtiéndose así el morfema - $n$ en un clítico y, por lo tanto, quedando sujeto a los mismos principios que los demás clíticos (principio de Wackernagel, concretamente), resulta también sumamente problemático para los contextos de primera persona del plural. En las páginas que siguen, procuramos destacar algunas observaciones que consideramos relevantes para avanzar en futuros análisis. Nuestra intención no es formalizar una propuesta, sino discutir posibles alternativas para pensar los análisis previos y desarrollar aspectos sobre la morfología de primera persona que podrían iluminar el abordaje de los datos.

Una alternativa para resolver el problema que supone la primera persona del plural en los abordajes previos es explorar la idea de que después de produ- 
cidas las operaciones propuestas en estos tres análisis, tenga lugar una operación de empobrecimiento (Halle, 1997) que borre la información de persona. Si el nodo AGR (o AGR transformado en clítico en términos de Arregi y Nevins) solo presenta la información de plural, el único ítem que puede insertarse es - $n$ y no -mos, que tiene también información de persona. Una propuesta como la de Kayne se salvaría asumiendo que cada nodo AGR que se agrega es defectivo $y$, por lo tanto, la única posibilidad es la ocurrencia de $-n$ como forma por defecto (o menos especificada). Esta solución daría lugar a un nuevo problema: se esperaría que - $n$ apareciera también con la segunda persona del singular. Si bien en nuestra búsqueda de datos y en ejemplos registrados en Heap y Pato (2012) encontramos algunos casos de este tipo, están todos restringidos a la ocurrencia de $-n$ con infinitivos y gerundios. No hemos encontrado casos de imperativo de segunda persona del singular (*cantálon; *decílon/*dílon).

(31) a. y si yo me siento bien haciendolon, no lo dejaré de hacer [Colombia] $<$ https://www.youtube.com/watch?v=QBTbs104zm4>

b. Gracias por pasar sigue haciendolon $<\mathrm{http}: / /$ www.fotolog.com/s_selena_g/50026000/>

c. pero tendré que curarmen las heridas de los pies [Adelgazar.net, 03/ $10 / 2006]^{19}$

d. Lo que si hago es apropiarmen de lo que me encuentro [Blog, 10/ 2006, M-19]

Para evitar el problema mencionado, se podría explorar la hipótesis de que la ocurrencia de $-n$ está motivada por rasgos que se copiaron de la estructura existente. Si se combina esta idea con la aplicación de una operación de empobrecimiento (Halle, 1997) se podría explicar la aparición de $-n$ cuando la información es 1PL. De manera informal, podríamos decir que en el contexto de $\mathrm{CL}\left(\right.$ ítico) $+\mathrm{AGR}_{[1 \mathrm{PL}]}$ el rasgo de persona [1] se borra y, por lo tanto, la inserción está motivada solo por [PL], de ahí que el copiado sea siempre con $-n$. Esta idea podría resultar interesante, ya que descarta tanto *demoslomos como *delomos; sin embargo, sigue dejando abierto el problema con respecto al desplazamiento: *delon, agramatical en la interpretación de primera persona del plural.

En resumen, si asumimos que - $n$ es un morfema que se inserta por defecto, sería esperable su aparición en contextos en los que no la encontramos (por ejemplo, en imperativos de segunda persona del singular). Por otro lado, si consideramos que $-n$ solo se vincula con [PL] y que cuando la información es [1PL] se produce el empobrecimiento del rasgo de persona, seguimos sin explicar los casos de desplazamiento, i. e. la inexistencia de *delon frete a delen.

19 (31c-d) son ejemplos extraídos de Heap y Pato (2012). 
Otra posibilidad que puede resultar iluminadora es considerar que el rasgo de número y el rasgo de persona en el morfema -mos, sean un poco más independientes de lo que se suele sostener. En esta línea, Harris (1995) propone una segmentación de los clíticos del español que podría resultar iluminadora. El autor sugiere que el clítico nos puede segmentarse en $n-O-s$, donde $n$ - se asocia al rasgo de primera persona, - $o$ - al marcador de clase por defecto (clase I según el autor) y $-s$ con número plural. Cada uno de estos elementos se inserta en un nodo independiente (remitimos a su trabajo para la discusión detallada). Considerando esta propuesta, podría pensarse que el morfema -mos se segmenta de manera similar, con lo que la información de persona estaría únicamente en $\mathrm{m}$-, mientras que $-s$ indicaría $[\mathrm{PL}]^{20}$. Si bien es cierto que esta propuesta se aleja de lo que se ha sugerido hasta ahora con respecto a la morfología de número y persona en español, habría evidencia empírica que permitiría avanzar en esta línea. De hecho, hay una serie de contextos en los que el morfema -mos no se realiza de la manera esperable.

(32) a. Vámonos> va-mo-nos (forma recomendada, frente a vamosnos)

b. comprémolos $>$ compré-mo-los ${ }^{21}$

(33) a. Los fuimos (por nos fuimos)

b. asi que pongamoslon las pilas

$<$ http://juguitodenaranjaa.blogspot.com.es/2007/08/estoy-calientesband.html>

Lo interesante de (32) es que parecería que la $-s$ de $-m o s$ tiene un estatuto relativamente independiente $\mathrm{y}$, como tal, su elisión es posible sin que se borre todo el morfema. Los datos de (33) correspondientes a hablantes de distintas variedades muestran también una independencia, en la forma del clítico. En (33a), lo que se ve alterado es el elemento que estaría asociado, de acuerdo a la segmentación de Harris, con la información de persona, mientras que en (33b) se ven alterados los dos: $l$ - por $m-\mathrm{y}-n$ por $-s$. En los casos que recopila De Benito (2015) habría un borramiento total de la información del nodo y el único elemento que se insertaría en ese contexto no especificado es se (se fuimos).

A estos datos podemos agregar evidencia interlingüística con respecto a los cambios sufridos por el morfema latino de 1PL -mus, en las lenguas romances. El catalán conserva para la primera persona del plural el morfema - $m$ (nosaltres cantem), algo que también sucede en rumano (noi dormim 'dormimos'), mientras que el italiano conserva -mo (noi cantiamo) ${ }^{22}$. Asimismo, Alvar y Pottier

\footnotetext{
${ }^{20}$ Minkoff (1993) llega a esta misma conclusión a partir de los datos del español del Caribe.

${ }^{21}$ Para la revisión de estos datos remitimos a Pato y De Benito (2017).

${ }^{22}$ Agradezco a Francesc Roca por esta observación.
} 
(1993: 194-195) señalan que en aragonés la desinencia latina -mus derivó en -nos, forma idéntica a la del clítico, en andaluz se perdió la $-s$ y en canario la $n$ - del pronombre y del clítico se cambia por $l$ - (losotros, los, vámolos), fenómeno que se observa también en el español rural de Argentina y Chile. Finalmente, la $m$ - propia de la desinencia verbal -mos se encuentra en judeo español en los pronombres y clíticos (mosotros, mos) (1993: 123). Lo que vemos es que el resultado del cambio permite pensar en una segmentación de este morfema y en una vinculación entre la $m$ - del sufijo flexivo y la $n$-que reconocemos en el pronombre y en el clítico ${ }^{23}$.

Si fuera posible, entonces, segmentar -mos en mo-s, donde $-s$ es [PL], entonces se explicaría de manera directa que el elemento que aparece en el fenómeno que nos compete sea $-n$, morfema vinculado en el ámbito verbal con la pluralidad. Además, nos permitiría dar cuenta de los datos agramaticales y vincular estos datos con otros que también involucran posiciones anómalas para la morfología de plural, como sucede con los plurales parasíticos (se los dije a los estudiantes).

Ahora bien, si esta segmentación fuera adecuada y lo que se copia es únicamente el rasgo asociado a la $-s$ de $-m o s, i$. e., [PL], deberíamos repensar las formas resultantes del desplazamiento en estos casos. Algo que hemos señalado es que el desplazamiento parece imposible en cualquiera de las variedades (*vendalon por vendamoslo; *vendalomos, etc.). Sin embargo, siempre lo hemos pensado en virtud de considerar el morfema -mos como un elemento no segmentable. Desde esta nueva perspectiva, deberíamos indagar por otras formas en las que solo se desplace el segmento con la información de plural. Lo esperable, entonces, sería que apareciera solo el morfema -mo-, porque el plural se desplazó al clítico y, dado que el plural en la flexión verbal se realiza como $-s$ solo en relación de localidad con la información de primera persona, deberíamos obtener la forma $-n$, que es el plural por defecto. Básicamente, la configuración por la que debemos indagar es verbo $+m o+\mathrm{CL}+n$, como paralela a verbo $+\mathrm{CL}+n$ (vendalon). Los datos de (34) muestran que esta predicción es correcta.

(34) a. El jugador uruguayo promedio es tremendo ladilla. Digamolon. $<$ https://twitter.com/soniabudassi/status/610976228435214336>

\footnotetext{
${ }^{23}$ Vale señalar, además, que ya en latín la $-m$ se reconocía como desinencia verbal relacionada con la primera persona en muchos de los tiempos: pretérito imperfecto de indicativo (amaba$m$ )/subjuntivo (amare-m); pretérito pluscuamperfecto (amavera- $m$ ); pretérito perfecto del subjuntivo (amaver-im); pretérito pluscuamperfecto del subjuntivo (amavisse-m), etc. Además, se reconoce esa desinencia en el presente del verbo essere (Ego su-m...). En todos los casos mencionados, se reconoce de manera independiente la morfología relacionada a tiempo/modo/aspecto, por lo que claramente el morfema $-m$ refiere a persona. Esta desinencia se pierde obedeciendo a un cambio fonético regular: pérdida de la $-m$ al final de palabra (v. Cano Aguilar, 1999: 152; Alvar y Pottier, 1993: 192; Penny, 1998: 154).
} 
b. yo vi esto en un foro donde jugaban 50 tipos en un solo topico sin problemas, no pasa nada che, hagamolon aca noma que somos menos de la mitad que esos

$<$ http://divididos.fr.yuku.com/topic/1452/Jugamon-al-PRODE-Clausura-2006\#.V_8yMfmLRpg>

c. Hagamolan este finde largo (en referencia a una salida en bicicleta) $<$ http://cbabikes.com.ar/salidas-eventos-encuentros/la-vuelta-al-lagoembalse-de-rio-3o-30102011-5570.270.html>

Vemos, entonces, que la consideración de todo el conjunto de datos de la primera persona del plural permite reconocer una posible línea de investigación para futuros análisis sobre el tema. Queda aún el desafío de intentar entender de qué manera se produce el copiado en las construcciones de infinitivo y gerundio, dado que, como se sigue de la sistematización que propusimos en $\S 2$, este fenómeno tiene lugar en construcciones muy diferentes, en las que las relaciones de localidad entre la proyección en la que se adjuntaría la morfología flexiva del verbo conjugado y la construcción de infinitivo no son las mismas en todos los casos. Consideramos que la sistematización realizada aquí puede ser una contribución válida para buscar respuestas a este fenómeno.

\section{RECAPITULACIÓN}

A lo largo de estas páginas, procuramos presentar un panorama lo más completo posible de la flexión verbal en posiciones inesperadas en español, fenómeno también conocido como pluralidad anómala (Heap y Pato, 2012), clonación de la flexión (Harris, 1995), metátesis de flexión verbal/copiado (Harris y Halle, 2005), reduplicación generalizada (Arregi y Nevins, 2012 y 2015). Asimismo, examinamos tres análisis (Arregi y Nevins, 2012 y 2015; Harris y Halle, 2005; Kayne, 2010) a partir de los datos que consideran, que, como mostramos, constituyen un subconjunto del panorama que describimos en $\S 2$. Asimismo, mostramos que muchos de los datos que expusimos suponen problemas importantes para estos análisis.

En nuestro panorama descriptivo, observamos que todas las posibilidades lógicas de combinar un verbo en imperativo con dos clíticos se registran en alguna variedad del español. También incluimos datos en los que el copiado se da con morfología de primera persona del plural. Estos datos no han sido considerados en análisis previos a nuestra presentación (Mare 2015) y resultan sumamente relevantes para revisar este fenómeno y, también, para repensar aspectos de la morfología verbal del español. Finalmente, propusimos una sistematización de tipos de construcciones de infinitivo y gerundio, formas verbales en las que también se registra este fenómeno. Un dato interesante que hemos 
recuperado es el de ascenso de clíticos, cuando el clítico se pronuncia también en la posición más baja y a este se le agrega la $-n$ inesperada (te están insultandoten). La ausencia de $-n$ en el clítico más alto es esperable y predecible en todos los análisis sobre este fenómeno. Sin embargo, este dato constituye un aporte para revisar la instancia en la que se produce el ascenso y el copiado. Evidentemente, el ascenso del clítico es anterior, de lo contrario, tanto a la izquierda como a la derecha, tendríamos la forma -ten.

Quedan aún varias cuestiones para continuar explorando, tanto desde un punto de vista descriptivo, como desde un abordaje teórico. Con respecto a la descripción, sería fundamental establecer una tipología del fenómeno, i. e., determinar en qué variedades se da cada una de las opciones señaladas, ya que como se ha indicado, no todas las opciones se observan en las mismas variedades. Desde el punto de vista teórico, consideramos que vale la pena avanzar en la exploración de propuestas que capten las intuiciones que expusimos en §4: las posiciones inesperadas, no serían "tan" inesperadas, sino que reflejarían la idea de que cierta morfología verbal debe aparecer al final de un ítem de carácter verbal. Las sugerencias que hemos realizado aquí intentan seguir esa línea. Esperamos, por tanto, que esta presentación constituya un aporte para avanzar con la comprensión de este fenómeno.

\section{BIBLIOGRAFÍA}

Acuña, Leonor y Andrea Menegotto (1998): "Dialecto y sistema pronominal del área mapuche", en Lucía Goluscio y Yosuke Kuramochi (comps.), Lingüística y literatura mapuche. Aproximaciones desde ambos lados de los Andes, Buenos Aires, Universidad Católica de Temuco/ Universidad de Buenos Aires, pp. 51-59.

Alvar, Manuel y Bernard Pottier (1993): Morfología histórica del español, Madrid, Cátedra.

Arregi, Karlos y Andrew Nevins (2012): Morphotactics: Basque auxiliaries and the structure of Spellout, Dordrecht, Springer.

Arregi, Karlos y Andrew Nevins (2015): "Morphotactic analysis and Spanish mesoclisis", presentación en el VII Encuentro de Gramática Generativa, Buenos Aires.

Belloro, Valeria (2015): To the Right of the Verb. An Investigation of Clitic Doubling and Right Dislocation in three Spanish Dialects, Cambridge, Cambridge Scholars Publishing.

Benito, Carlota de (2015): "Pero se escondíamos como las ratas: syncretism in the reflexive paradigm in Spanish and Catalan", Isogloss, 1/1, pp. 95-127.

Bonet, Eulàlia (1995): "Feature structure of Romance clitics", Natural Language and Linguistic Theory, 13, pp. 607-647.

Cano Aguilar, Rafael (1999): El español a través de los tiempos, Madrid, Arco/Libros.

Colantoni, Laura y M. Cristina Cuervo (2013): "Clíticos acentuados", en Laura Colantoni y Celeste Rodríguez Louro (eds.), Perspectivas teóricas y experimentales sobre el español de la Argentina, Madrid/Frankfurt, Iberoamericana/Vervuert, pp. 143-158.

Company Company, Concepción (1992): "Un cambio en proceso: «El libro, ¿quién 'se los' prestó?»", en Elizabeth Luna (ed.), Scripta philologica in honorem Juan M. Lope Blanch, México, Universidad Nacional Autónoma de México, pp. 349-363. 
COSER = Inés Fernández-Ordóñez (dir.) (2005-): Corpus Oral y Sonoro del Español Rural, $<$ www.corpusrural.es $>$.

Halle, Morris (1997): "Distributed Morphology: Impoverishment and Fission", MIT Working Papers in Linguistics, 30, pp. 425-449.

Halle, Morris (2008): "Reduplication", en Robert Freidin, Carlos P. Otero y María Luisa Zubizarreta (eds.), Foundational issues in linguistic theory: Essays in honor of Jean-Roger Vergnaud, Cambridge, Massachusetts Institute of Technology Press, pp. 325-357.

Halle, Morris y Alec Marantz (1994): "Some Key Features of Distributed Morphology", en Andrew Carnie, Heidi Harley y Tony Bures (eds.), Papers on Phonology and Morphology, Cambridge, Massachusetts Institute of Technology Press, pp. 275-288.

Harris, James (1991): "With respect to accentual constituents in Spanish", en Hector Campos y Fernando Martínez-Gil (eds.), Current studies in Spanish linguistics, Washington D.C., Georgetown University Press, pp. 447-473.

Harris, James (1995): "The morphology of Spanish clitics", en Hector Campos y Paula Kempchinsky (eds.), Evolution and revolution in linguistic theory: Essays in honor of Carlos Otero, Washington D.C., Georgetown University Press, pp. 168-197.

Harris, James y Morris Halle (2005): "Unexpected Plural Inflections in Spanish: Reduplication and Metathesis", Linguistic Inquiry, 36/2, pp. 195-222.

Heap, David y Enrique Pato (2012): "Plurales anómalos en los dialectos y en la historia del español", en Emilio Montero Cartelle y Carmen Manzano Rovira (eds.), Actas del VIII Congreso Internacional de Historia de la Lengua Española, Santiago de Compostela, Asociación de Historia de la Lengua Española/Meubook, vol. I, pp. 829-840.

Kany, Charles (1994 [1945]): Sintaxis hispanoamericana, Madrid, Gredos.

Kayne, Richard (1992): "Word Order", presentación en el Coloquio Generative Linguistics in the Old World, GLOW, Lisboa, 14 de abril.

Kayne, Richard (2010): "Toward a syntactic reinterpretation of Harris y Halle (2005)", en Reineke Bok-Bennema, Brigitte Kampers-Manhe y Bart Hollebrandse (eds.), Romance Languages and Linguistic Theory 2008: Selected papers from "Going Romance" Groningen 2008, Amsterdam, John Benjamins, pp. 145-170.

Malvestiti, Marisa (1993): "Castellano mapuchizado en la línea Sur. Aproximaciones", en Actas de las Primeras Jornadas de Etnolingüística, Rosario, Universidad Nacional de Rosario, pp. 137-144.

Manzini, Rita y Leonardo Savoia (2011): "Mesoclisis in the Imperative: Phonology, Morphology or Syntax?", Lingua, 121, pp. 1101-1120.

Mare, María (2013): "La concordancia comitativa en el español rioplatense", en Angela di Tullio (coord.), El español de la Argentina: estudios gramaticales, Buenos Aires, Eudeba, pp. 59-84.

Mare, María (2015): Proyecciones funcionales en el ámbito nominal y concordancia. Un abordaje en términos de variación, Edición lingüística 97, Múnich, Lincom.

Minkoff, Seth (1993): "Plurality, Clitics, and Morphological Merger in Caribbean Spanish", en Vern M. Lindblad y Michael Gamon (eds.), Papers from the $5^{\text {th }}$ Student Conference in Linguistics, MIT Working Paper in Linguistics 20, Cambridge, MIT, pp. 177-192.

Ordóñez, Francisco (2014): "Clitics in Spanish", en José Ignacio Hualde, Antxon Olarrea y Erin O'Rourke (eds.), The Handbook of Hispanic Linguistics, Oxford, Wiley Blackwell, pp. 423-452.

Pato, Enrique y Carlota de Benito (2017): "Tráenolos para comernolos o la «transposición» del clítico en español actual", Philologica Jassyensia, 1, 25, pp. 121-136.

Penny, Ralph (1998): Gramática histórica del español, Barcelona, Ariel Lingüística.

Roca, Iggy (1986): "Secondary stress and metrical rythm", Phonology Yearbook, 3, pp. 341-370.

Fecha de recepción: 24 de octubre de 2016

Fecha de aceptación: 19 de enero de 2017 